\title{
Skin Response Numeric Result in Standard Unit
}

National Cancer Institute

\section{Source}

National Cancer Institute. Skin Response Numeric Result in Standard Unit. NCI

Thesaurus. Code C117682.

The numerical identifier of skin response result in standard units. 